\title{
Researching Context-Aware Recommendation Systems in Mobile Environments
}

\author{
María del Carmen Rodríguez-Hernández, Sergio Ilarri \\ Distributed Information Systems (SID) \\ Instituto de Investigación en Ingeniería de Aragón (I3A). \\ Universidad de Zaragoza, Mariano Esquillor s/n, 50018, Zaragoza, Spain. \\ Tel. +34-976762707, Fax +34-976762043, e-mail: 692383@unizar.es
}

\begin{abstract}
The design and implementation of generic frameworks to support an easy development of context-aware recommendation systems has been relatively unexplored. In this paper, we briefly present our ongoing work for the definition of a framework that will support the development of context-aware recommendation systems in distributed and mobile environments. Those systems will allow suggesting relevant items to mobile users.
\end{abstract}

\section{Introduction}

Recommendation Systems (RS) are applications which suggest (relevant) items to users. The suggestions can help to solve certain decisionmaking problems which are presented to the users, such as which books to buy, which movies to watch, or which online news to read. They try to adapt the suggestions to each user individually, based on his/her preferences. However, traditional RS are not designed for mobile environments, where the context (e.g., the location, the time, the weather, the presence of other people, etc.) and the movements of the users may be important factors to obtain relevant and helpful recommendations.

The emergence of Context-Aware Recommender Systems (CARS) has prompted the growth of recommendation algorithms that incorporate context information. Indeed, Adomavicius and Jannach claimed recently that there is still much research needed in the field of CARS [2]. They emphasized that the design of flexible and generic architectures as well as frameworks to support an easy development of CARS remains quite unexplored.

Based on the above issues, we focus on the following scientific problem: how could we facilitate the development of CARS in distributed environments to provide mobile users with relevant recommendations? So, the object of our research study is the process of context-aware recommendation for mobile users. Specifically, we pursue the following general goal: the development of a mobile context-aware framework which will allow providing relevant recommendations to mobile users. We aim at generic mobile computing scenarios where different communication technologies (e.g., 3G, Wi-Fi), interaction protocols (centralized approaches vs. mobile peer-to-peer), and data access modes (push-based data access, pull-based data access, and hybrid data access) may be available. Moreover, the relevant data used as a basis for the recommendations may be available locally on the mobile device, centralized on a server, or distributed among different data sources (mobile devices and other static nodes). The recommendation framework should thus adapt itself to different typical mobile computing scenarios.

\section{Related Work}

Three context-aware recommendation paradigms were proposed in [1]: pre-filtering, where the contextual information is used to filter the data set before applying traditional recommendation algorithms; post-filtering, where the ratings are predicted using a conventional RS, taking all the input data available into account, and then the resulting set of recommendations is adjusted (contextualized) for each user by using contextual information; and contextual modeling, which uses the context information directly in the modeling technique.

Particularly in mobile environments, where the user is moving and the context is highly dynamic, it is important to provide precise recommendations and avoid over loading the user with the suggestion of many items. Therefore, a recommendation system should try to relieve the user from having to type or introduce significant information as an input, favoring implicit recommendations (based on the context and user preferences) over explicit (querybased or user-initiated) recommendations. Along these lines, [4] proposes a proactive recommender system that pushes recommendations to the user when the current situation (i.e., the context) is 
considered appropriate, without explicit user requests.

Recently, a software framework called Hybreed [3] has been presented for building complex contextaware applications. The framework is based on a quite generic notion of context. However, the authors identified elements pending for future work.

Despite these interesting proposals, existing work has not focused so far on mobile computing scenarios. So, there is a need to define a generic and abstract framework for the modeling of CARS in mobile environments. With our work, we attempt to bring recommendation systems to dynamic mobile computing environments.

\section{Goals and Approach}

To accomplish our goal, the first step is to perform a detailed study of the state of the art on contextaware recommendation systems, as well as an analysis of existing frameworks, which may partially solve some of the problems arising in this field. The next step is to develop an appropriate and general architecture that supports context-aware recommendations in generic mobile environments. Finally, we will evaluate and validate the proposed framework by considering several application scenarios.

The ultimate goal of this research is to develop a framework that will provide relevant context-aware recommendations for mobile users. We will also probably need to develop a suitable simulator that will allow representing mobile users that receive real-time context-aware recommendations in both indoor and outdoor scenarios. An intermediate contribution will be the design of a generic architecture to support an easy development of CARS for mobile users. Overall, with this research, we hope to contribute to bridge the gap between the fields of recommendation systems and mobile computing.

\section{Current Status and Next Steps}

So far, we have performed an extensive study of the state of the art on CARS. We have identified that most of the related research focuses on the problem of understanding and including contextual information in the recommendation process. However, the design of generic architectures and frameworks to support an easy development of CARS has received little attention, especially in mobile environments.

We have also started the development of a generic context-aware mobile recommendation architecture. It will include several traditional recommendation algorithms (e.g., based on collaborative filtering and content-based), as well as hybrid algorithms that combine several of them to try to improve the accuracy of the recommendations. Furthermore, it will provide different context-aware recommendation paradigms (e.g., pre-filtering, postfiltering, and contextual modeling). The recommendation process will consider both static context information obtained from the user's profile as well as dynamic context data obtained from the environment (e.g., by using sensors). Moreover, it will support the exploitation of other external data sources available, such as geospatial information services, social networks, and web services.

The user will be able to receive both proactive recommendations (real-time recommendations of items without explicit requests from the user) and reactive recommendations (obtained as an answer to a query submitted by the user and evaluated by the system as a continuous query). Different types of environments, including outdoor environments (e.g., roads, pedestrian paths) and indoor environments (e.g., a mall, a hotel), will be supported.

\section{REFERENCES}

[1]. ADOMAViciUS, G. and TUZHILIN, A. ContextAware Recommender Systems. In: RICCI, F. et al. Recommender Systems Handbook. New York: Springer, 2011, pp. 217-253.

[2]. ADOMAVICIUS, G. and JANNACH, D. Preface to the Special Issue on Context-Aware Recommender Systems. User Modeling and User-Adapted Interaction. 2014, 24(1-2), 1-5. Available from: doi:10.1007/s11257-013-9139-2.

[3]. HUSSEIN, T. et al. Hybreed: A Software Framework for Developing Context-Aware Hybrid Recommender Systems. User Modeling and User-Adapted Interaction. 2014, 24(1-2), 121-174. Available from: doi:10.1007/s11257-012-9134-z.

[4]. WOERNDL, W. et al. A Model for Proactivity in Mobile, Context-Aware Recommender Systems. In: MOBASHER, B. et al., eds. Proceedings of the ACM International Conference on Recommender Systems. New York: ACM, 2011, pp. 273-276. 